\title{
El sexo del cerebro: entre ciencia e ideología
}

\author{
Catherine Vidal $^{1}$
}

1. Neurobióloga, Directrice de recherche honoraire à l'Institut Pasteur de Paris, Miembro del Comité d'Ethique de l'Institut National de la Santé et de la Recherche Médicale (INSERM) y co-responsable del grupo «Genre et Recherches en Santé».

Autor correspondiente: Catherine Vidal, E-mail: cvidal75005@gmail.com

\begin{abstract}
Resumen
A pesar de los avances en el conocimiento en neurociencias, los prejuicios y los estereotipos sobre las diferencias en las habilidades y el comportamiento entre los sexos todavía están vivos en el espacio público. Los medios de comunicación e Internet nos inundan con viejos clichés que afirman que las mujeres son naturalmente dotadas para la empatía pero incapaces de leer un mapa de rutas, mientras que los hombres son esencialmente buenos en matemáticas y competitivos. Estos discursos sugieren que nuestras habilidades y nuestras personalidades están programadas en el cerebro y son inmutables. Investigaciones recientes muestran por el contrario que, gracias a sus formidables propiedades de «plasticidad», el cerebro produce constantemente nuevos circuitos de neuronas de acuerdo con el aprendizaje y las experiencias de la vida. El concepto de plasticidad cerebral es esencial para abordar la cuestión del origen de las diferencias y similitudes entre los sexos y aporta una luz neurobiológica fundamental sobre los mecanismos que participan en la construcción de nuestras identidades de mujeres y hombres. De este modo, consolida y enriquece la investigación en ciencias humanas sobre género.
\end{abstract}

Palabras clave: Género - Sexo - Plasticidad cerebral - Neuroimágenes cerebrales IRM - Neuroética.

\section{GENDER IN THE BRAIN: BETWEEN SCIENCE AND IDEOLOGY}

\begin{abstract}
Despite tremendous advances in neurosciences, the idea that biology is a major determinant for gender differences in cognition and behavior, is still alive. Media and internet continue to feed the public with old clichés that claim that women are naturally good for empathy but unable to read a map, while men have a brain suited for maths and competition. These discourses suggest that at birth the intellectual abilities are wired differently in the brains of girls and boys. Such a view is in total contradiction with today's knowledge on cerebral plasticity, and on the role of the environment in re-shaping the brain through learning and experiences. The plasticity concept allows new insight to the question of the origin of brain differences and similarities between the sexes. Experience in the sociocultural context involves gender learning processes which interact with the biological processes. A major challenge is that of building an interdisciplinary dialogue across the biological, social, and human sciences to develop new approaches linking sex, gender and the brain.
\end{abstract}

Key words: Gender - Sex - Cerebral plasticity - Brain imaging MRI - Neuroethics. 
"Pretender separar lo biológico de lo cultural no tiene sentido. Sería lo mismo que preguntar, si la atracción de Romeo por Julieta es de origen genético o cultural"

François Jacob, premio Nobel de Fisiología. (Le jeux des possibles, 1981).

Entre las grandes representaciones que la humanidad tiene del mundo, la diferencia de los sexos es una base fundamental. La polaridad hombre/mujer aparece como evidencia elemental, «un tope final para el pensamiento» según la fórmula de Françoise Héritier (20). El clivaje entre los sexos elaborado por filósofos, teólogos, historiadores y naturalistas de siglos pasados es visto por muchos como un reflejo de una naturaleza humana eterna.

Actualmente, la dualidad de las diferencias entre los sexos, en tanto evidencia natural, ha sido cuestionada por los estudios de género y numerosas investigaciones en el área de las humanidades y de las ciencias sociales. En el campo de las neurociencias, el descubrimiento de la «plasticidad cerebral» arroja una luz fundamental sobre los procesos de construcción social y cultural de las identidades de género $(43,45)$. Gracias a las técnicas de imágenes cerebrales por MRI (imágenes cerebrales por resonancia magnética), ahora sabemos que el cerebro está construido en interacción con el mundo circundante: se crean nuevas conexiones entre las neuronas a lo largo de la vida en función de las experiencias y el aprendizaje. El concepto de plasticidad cerebral es una verdadera revolución en nuestras concepciones del funcionamiento del cerebro humano. Nada está fijado para siempre en el cerebro desde el nacimiento, cualquiera sea el sexo y la edad.

Con estos avances científicos, uno estaría tentado a creer que los prejuicios y los estereotipos sobre las diferencias en las habilidades y el comportamiento entre los sexos se han eliminado. Sin embargo, claramente, ese no es el caso en la realidad cotidiana. La televisión, los sitios web, los medios gráficos, afirman que las mujeres son naturalmente multitareas, dotadas para la empatía pero incapaces de leer un mapa de rutas, por ejemplo; mientras que los hombres son esencialmente buenos en matemáticas, competitivos y pendencieros. Pero los medios no son los únicos involucrados. Ciertos círculos científicos también ayudan a promover la idea de un determinismo biológico innato de las diferencias en las aptitudes y los roles sociales entre los sexos. Estas tesis alimentan corrientes conservadoras que se oponen a la diversidad social y política, la igualdad de derechos para las mujeres, para las personas homosexuales, etc.

Este artículo tiene como objetivo aportar elementos de comprensión de los debates científicos actuales sobre el cerebro, el sexo y el género. El concepto de plasticidad cerebral es esencial para abordar la cuestión del origen de las diferencias y similitudes entre los sexos. Concluiremos con una reflexión ética frente a las variaciones en la interpretación de ciertos estudios científicos por resonancia magnética que conducen a reforzar los este- reotipos sobre las diferencias en las habilidades y el comportamiento entre los sexos. El tema del sexo cerebral nunca es neutral.

\section{Diferencias entre los sexos y plasticidad cerebral}

Cómo podemos responder hoy a la pregunta: ¿el cerebro tiene un sexo? La respuesta científica es sí y no. Sí, porque el cerebro controla las funciones fisiológicas asociadas con la reproducción sexual. Pero, con respecto a las funciones cognitivas, el conocimiento actual sobre el desarrollo del cerebro y la plasticidad cerebral muestra que las niñas y los niños, las mujeres y los hombres, tienen el mismo potencial de razonamiento, memoria, atención, imaginación, etc.

\section{La construcción del cerebro de niñas y niños}

Al nacer, los niños son en promedio más pesados y más altos que las niñas. Su volumen cerebral es aproximadamente un 10\% más grande que el de las niñas. Si se relaciona el volumen del cerebro con el tamaño del cuerpo, la diferencia entre los sexos es del 6 al 8\% y se mantiene significativa. Con respecto a la estructura interna del cerebro, varios estudios de resonancia magnética han mostrado variaciones según el sexo en los volúmenes de materia gris (donde se concentran los cuerpos celulares de las neuronas) y la sustancia blanca (que consiste en fibras nerviosas de los cuerpos celulares. Desde el nacimiento hasta la edad adulta, las niñas tienen en promedio un poco más de materia gris y los niños un poco más de materia blanca $(7,15,16,18)$.

Esas diferencias cerebrales han dado lugar a todo tipo de especulaciones que supuestamente explican las diferencias entre los sexos en lo atinente a la orientación espacial, el razonamiento, la intuición, etc. Sin embargo, estudios recientes han cuestionado la interpretación de las diferencias anatómicas entre los cerebros de mujeres y hombres. Resulta que las diferencias en cuestión son solo aparentes. Desaparecen si tenemos en cuenta el tamaño del cerebro como tal $(8,18,23,32,34,40)$. Así, cuando comparamos los cerebros de hombres y mujeres del mismo volumen (15 a $20 \%$ de la población), ya no se ven diferencias en las proporciones de materia gris y blanca. Es el tamaño del cerebro y no el sexo lo que explica las diferencias en la proporción de las materias gris y blanca. Esta observación muestra que el desarrollo del cerebro difiere según el espacio disponible en el cráneo y esto no tiene ningún impacto en las funciones intelectuales. Por lo tanto, entendemos que los estudios que comparan los cerebros de los dos sexos sin tener en cuenta el tamaño del cerebro (este sigue siendo el caso para la mayoría de los estudios) pueden llevar a conclusiones erróneas sobre el origen de las diferencias cognitivas entre los sexos. Un remarcable estudio sobre el desarrollo y la maduración del cerebro de 3000 sujetos de entre 9 meses y 94 años de edad fue realizado en 2017 (6). La resonancia magnética mostró en ese trabajo que la estructura de las diferentes regiones cerebrales es 
globalmente idéntica para los dos sexos si se tiene en cuenta el volumen del cerebro. Solamente se observaron diferencias menores a partir de los 80 años de edad.

Queda mucha investigación por hacer sobre las interacciones mutuas entre los factores ambientales y los procesos de desarrollo en los niños pequeños. Estas preguntas son cruciales para determinar el origen del lenguaje y los trastornos del comportamiento como la dislexia, la hiperactividad o el autismo, que afectan más a los niños que a las niñas. Una de las claves para comprender estos mecanismos es el estudio de la plasticidad cerebral.

\section{Hormonas sexuales y desarrollo del cerebro}

Durante la vida intrauterina, el feto humano baña en un entorno hormonal que varía según el sexo. Entre las 14 y las 20 semanas de gestación, las mediciones realizadas en el líquido amniótico muestran que éste contiene el doble de testosterona para un feto masculino que para un feto femenino. En contraste, en los fetos femeninos, la hormona folicular FSH está siete veces más concentrada que en los hombres. Los niveles de estrógeno son equivalentes en ambos sexos. Después del nacimiento, los bebés tienen altos niveles de testosterona en la sangre hasta los dos meses de edad. Luego, la testosterona cae gradualmente hasta los siete meses de edad y permanece en niveles muy bajos para volver a remontar en la pubertad (10).

La acción de estas hormonas en el cuerpo es bien conocida. En fetos masculinos, la alta concentración de testosterona induce la masculinización de los genitales. En fetos femeninos con dosis bajas de testosterona, el tracto genital adquiere una estructura femenina.

Las hormonas sexuales también actúan sobre el cerebro de los fetos, especialmente en la región del hipotálamo, la cual, desde la pubertad, controla las funciones reproductivas. Así, en los cerebros de las mujeres, las neuronas del hipotálamo se activan cada mes para que la glándula pituitaria secrete las hormonas LH y FSH que desencadenarán la ovulación. En los hombres, las neuronas del hipotálamo tienen una acción continua, no cíclica, sobre la liberación de LH y FSH, que estimulan la producción de testosterona y la producción de espermatozoides en los testículos.

Con respecto a la acción de las hormonas sexuales sobre el desarrollo cognitivo y el comportamiento, la investigación actual ha desafiado en gran medida las viejas teorías. Los biólogos han creído durante mucho tiempo que las diferencias de comportamiento entre los sexos están relacionadas con la exposición fetal a la testosterona (26). Según la teoría de «organización/activación» del cerebro lanzada hace 50 años, la acción de la testosterona en el cerebro del feto masculino deja una huella indeleble responsable de las diferencias entre los sexos en muchos campos: sexualidad, habilidades cognitivas, personalidad, intereses, emociones, trastornos mentales, etc.

Uno de los problemas con esta teoría es que no es verificable. De hecho, es imposible medir la testosterona en el cerebro de los fetos humanos para demostrar que, según su concentración, los comportamientos futuros de los niños serán más o menos «masculinos» o «femeninos». Sin embargo, existe una situación patológica que puede proporcionar pistas. Es una malformación de las glándulas suprarrenales del feto que producen cantidades excesivas de testosterona (hiperplasia suprarrenal congénita o CAH). Como las bebés nacen con genitales masculinizados, se planteó la pregunta de si sus cerebros y comportamientos también serían masculinizados. La respuesta es no, como se muestra en una revisión reciente que consideró más de cien estudios sobre este tema (27). Primero, los cerebros de las niñas con CAH no son anatómicamente diferentes de los de las demás. Algunos estudios han informado comportamientos «atípicos» en algunas pacientes. Sin embargo, debe tenerse en cuenta que estas jóvenes se han sometido desde su infancia a cirugías repetidas de los órganos genitales y urinarios para feminizarlas y estos actos invasivos y dolorosos no están exentos de consecuencias sobre el desarrollo psicológico con respecto a la sexualidad. Además, el hecho de nacer con genitales masculinos despierta una mirada particular de los padres y familiares y tiene repercusiones en las relaciones sociales en general. Este contexto específico podría explicar ciertos comportamientos de las niñas con CAH que jugarían con más frecuencia con los juguetes de varones (construcción, automóviles) que otras niñas.

Durante la última década, se ha acumulado evidencia experimental para demostrar que la teoría de que la exposición fetal a la testosterona explica las diferencias en el comportamiento entre los sexos no tiene consistencia científica $(13,26,28)$. Además, esta teoría se basa en una concepción rígida del funcionamiento del cerebro desde el nacimiento. Esta perspectiva está en completa contradicción con el conocimiento actual sobre plasticidad cerebral que muestra cómo el aprendizaje y las experiencias de la vida dan forma al cerebro, tanto en niños como en adultos.

\section{La plasticidad cerebral}

Cuando nace el recién nacido, su cerebro tiene cien mil millones de neuronas, que dejan de multiplicarse. Pero la construcción del cerebro está lejos de terminar, ya que las conexiones entre las neuronas, las sinapsis, apenas comienzan a formarse: solo el 10\% de ellas están presentes al nacer. Esto significa que el 90\% de las sinapsis se hacen desde el momento en que el bebé comienza a entrar en contacto con el mundo exterior. ¡Se estima que en un cerebro adulto cada neurona está conectada a otras diez mil, lo que corresponde a un millón de billones de sinapsis! Sin embargo, solo seis mil genes están involucrados en la construcción del cerebro. Claramente, esto no es suficiente para controlar la formación de miles de millones de conexiones. El destino de nuestras neuronas no es parte del programa genético (38). La influencia del medio ambiente (físico, emocional, social, cultural, etc.) juega un papel importante en el cableado de las neuronas y la construcción del cerebro. 
El sistema visual es una ilustración vívida de esto. Al nacer, la visión del bebé es muy básica. Es solo a la edad de 5 años que los niños tienen capacidades visuales comparables a las de los adultos (2). Por lo tanto, lleva 5 años llevar a cabo el cableado de las neuronas que transportan información visual desde la retina y el nervio óptico hasta la corteza cerebral adonde se analizan las señales luminosas. Por lo tanto, es necesario que el impacto de la luz en la retina como una condición esencial para que las neuronas visuales se conecten correctamente. La falta de estimulación visual que padecen los niños con cataratas puede conducir a la ceguera (1).

El advenimiento de las técnicas de imagen cerebral ha sido crucial para revelar el papel de la plasticidad cerebral en el desarrollo del cerebro y en los procesos de aprendizaje $(3,39,43,45)$. Por ejemplo, en los pianistas, hay un engrosamiento de las regiones de la corteza cerebral especializadas en el movimiento de los dedos y en la audición. Este fenómeno se debe a la producción de conexiones adicionales entre las neuronas. Además, estos cambios en la corteza son directamente proporcionales al tiempo dedicado al aprendizaje del piano durante la infancia. Aprender conceptos abstractos también puede cambiar la estructura del cerebro. En los matemáticos profesionales, las regiones involucradas en el cálculo y la representación geométrica están engrosadas. Otro ejemplo elocuente de plasticidad cerebral se ha descrito en sujetos que aprenden a hacer malabarismos con tres bolas. Después de tres meses de práctica, la resonancia magnética muestra un engrosamiento de las regiones especializadas en visión y coordinación de movimientos de brazos y manos. Y si la capacitación se detiene, las mismas áreas se reducen (33).

Estos ejemplos, y muchos otros, muestran cómo la propia historia de cada persona está inscripta en su cerebro. Como resultado, ningún cerebro se parece a otro, incluidos los de gemelos. Algunos estudios de resonancia magnética muestran diferencias entre los cerebros de mujeres y hombres. Estos experimentos se han llevado a cabo principalmente en unas pocas docenas de individuos. Pero cuando las comparaciones se relacionan con cientos de personas, se borran las diferencias que podrían haberse observado en un pequeño número de sujetos. De hecho, las diferencias cerebrales entre personas del mismo sexo son tan grandes que, con frecuencia, superan las diferencias entre los sexos. Esta gran diversidad de cerebros explica por qué cuando hacemos promedios estadísticos no encontramos ningún signo anatómico o funcional específico para cada sexo $(24,25,29)$. Tal resultado muestra que la experiencia individual prevalece sobre un posible determinismo biológico ligado al sexo (genético u hormonal), pero también sobre un condicionamiento social de género durante la infancia que no quede inscripto en el cerebro.

El concepto de plasticidad permite superar el clásico dilema que tiende a oponer la naturaleza y la cultura. De hecho, lo innato y lo adquirido son inseparables ya que la interacción con el medio ambiente es la condición esencial para el desarrollo y funcionamiento del cere- bro. Lo innato aporta la capacidad de cableado entre las neuronas, lo adquirido permite la realización efectiva de este cableado. Estos avances en el conocimiento neurobiológico consolidan y enriquecen la investigación en humanidades y ciencias sociales sobre género. Aunque no le plazca a ciertos círculos conservadores, el género no niega la realidad biológica, por el contrario, la integra. El sexo y el género no son variables separadas, sino que se articulan en un proceso denominado de incorporación («embodiment»); término que designa la interacción entre el sexo biológico y el entorno social, y esto desde el nacimiento $(9,12,30,31)$.

\section{El neuro-sexismo en las publicaciones científicas}

Desde la aparición de la neurobiología, la cultura y la ideología han permeado la investigación sobre las diferencias entre el cerebro femenino y el masculino. En el siglo XIX, la forma del cráneo y el tamaño del cerebro se utilizaron para justificar la jerarquía entre los sexos (17). Se creía que los hombres, supuestamente más inteligentes, naturalmente tenían cerebros más grandes que los de las mujeres. Algunos médicos, en particular Paul Broca, han alimentado estas tesis con mediciones comparativas de cerebros cuidadosamente seleccionados para apoyar su demostración. Aunque al mismo tiempo otros estudios habían demostrado claramente que el tamaño del cerebro no era la causa de la inteligencia, la ideología de la burguesía conservadora prevaleció sobre el rigor científico. La interferencia entre la ideología naturalista y la práctica científica sigue siendo válida en el siglo XXI. En neurociencias, no es raro ver publicaciones en las que se utilizan imágenes del cerebro para justificar las diferencias de género basadas en un determinismo biológico. La resonancia magnética no escapa al «neuro-sexismo» $(14,21,36,37,41,42,43)$. Los siguientes ejemplos ilustran esto.

\section{¿El juicio moral de las mujeres es menos fiable que el de los hombres?}

La resonancia magnética se ha utilizado recientemente para comparar las habilidades de juicio moral entre los sexos (19). El experimento consistió en analizar los cerebros durante la presentación de fotos que ilustran escenas donde se socavaba «el orden moral»: crímenes, violencia física, altercados, etc. Las imágenes de los cerebros de las mujeres mostraron activaciones de regiones vinculadas a las emociones, mientras que las zonas activadas en los hombres estaban involucradas en la cognición. Los autores del estudio concluyeron que la resonancia magnética «confirma la diferencia de género en el juicio moral, con las mujeres centradas en el cuidado de los demás y la empatía, y los hombres en la evaluación racional de las reglas de justicia». ¡Por lo tanto, esto daría una prueba científica de que los hombres estarían dotados de un sentido moral mucho más confiable que el de las mujeres!

Este ejemplo de deriva sexista en la interpretación 
de un experimento por resonancia magnética reenvía al problema de la autoridad de los expertos científicos ante una audiencia no científica $(5,35)$. ¿Cómo estimar la validez de los argumentos avanzados cuando uno no es un especialista? Para frustrar las interpretaciones abusivas, es necesario tener en cuenta los métodos y procedimientos utilizados en MRI:

- Las condiciones de la experiencia para probar el juicio moral no tienen nada que ver con la realidad de la vida. La persona que yace en el aparato de resonancia magnética solo está mirando imágenes. Debe permanecer estrictamente estacionaria durante varias decenas de minutos, sin hablar, tragar o parpadear, con el riesgo de perturbar la adquisición de datos. Todo eso en un entorno de sonido parecido al de un martillo neumático...

- El experimento se llevó a cabo en un pequeño número de sujetos, 14 mujeres y hombres, lo que limita la importancia estadística de los resultados y su generalización.

- Finalmente, es importante tener en cuenta que la resonancia magnética solo proporciona una instantánea del estado del cerebro de una persona en un instante t. Dada la plasticidad cerebral, ver las diferencias entre los sexos en los «circuitos de la moralidad» no significa que reflejen predisposiciones innatas, como lo interpretan los autores del artículo. Las experiencias de vida y las normas sociales por sí solas pueden explicar las diferencias en la actividad cerebral entre los sexos observados en la pequeña muestra de personas analizadas. Esta alternativa ni siquiera fue mencionada por los autores...

\section{¿El cerebro de los hombres está conectado para la acción y el de las mujeres para la intuición?}

Según los comentarios de los mass media en enero de 2014, los investigadores estadounidenses han descubierto diferencias en las conexiones cerebrales entre los sexos que se cree que son responsables de las diferencias de comportamiento entre mujeres y hombres (22). Sin embargo, los datos experimentales presentados en la publicación científica están lejos de autorizar las conclusiones anunciadas $(11,18,32)$.

El estudio en cuestión utilizó una técnica específica de resonancia magnética (tensor de difusión) para analizar las fibras nerviosas que conectan diferentes regiones del cerebro. Las imágenes presentadas en el artículo muestran diferencias sorprendentes: los hombres tienen conexiones dentro del mismo hemisferio, mientras que las mujeres tienen conexiones entre los dos hemisferios. Estas imágenes son solo representaciones estadísticas de probabilidades de conexión y no corresponden a la anatomía real. Los hombres obviamente tienen fibras nerviosas entre los dos hemisferios y las mujeres en el mismo hemisferio. Sin embargo, los autores del artículo eligieron una ilustración sesgada de sus resultados que da la ilusión de una diferencia radical entre los cerebros de mujeres y hombres. Estas imágenes han sido amplia- mente utilizadas por los medios de comunicación como una prueba «objetiva» de las diferencias naturales entre los sexos.

Otro sesgo importante de este estudio radica en la interpretación de los datos. Según los autores, sus resultados sugieren que "los cerebros masculinos están estructurados para facilitar la coordinación entre la percepción y la acción, mientras que los cerebros femeninos están diseñados para facilitar la comunicación entre el análisis y la intuición". Esta interpretación es puramente especulativa. Los datos presentados en la publicación se refieren solo a la anatomía de las conexiones cerebrales, sin correlaciones con las pruebas de comportamiento que se llevaron a cabo simultáneamente. Por otro lado, debe recordarse que el estado actual del conocimiento del cerebro humano no permite establecer relaciones de causa y efecto entre la estructura anatómica del cerebro y el comportamiento.

Finalmente, es al menos sorprendente que la cuestión fundamental del origen de las diferencias en las conexiones entre los sexos no se haya abordado en la discusión de los resultados. El concepto de plasticidad cerebral ni siquiera se menciona. Sin embargo, sabemos que la mayoría de las conexiones se realizan después del nacimiento en función del aprendizaje y el entorno sociocultural. Los autores del artículo ignoraron la dimensión de género y centraron su argumentación en los «roles complementarios de hombres y mujeres en la procreación y la sociedad». Los términos de su conclusión son claros: «Nuestros resultados confirman que la complementariedad de los comportamientos entre los sexos proviene de un sustrato neural».

Esta publicación constituye un «caso típico» de validación pseudocientífica de los estereotipos de género. Es sorprendente que este artículo, cuya calidad científica es cuestionable, haya sido aceptado para su publicación en la prestigiosa revista estadounidense Proceedings of the National Academy of Sciences. Los forzamientos de los criterios para seleccionar artículos no son infrecuentes tan pronto como tocamos temas que afectan a la sociedad y que son "perfectos" para tener una amplia difusión en los medios de comunicación, con la consecuente notoriedad asegurada para los científicos y editores de revistas. El tema del sexo cerebral es ejemplar a este respecto.

Finalmente, esta publicación recibió varios desmentidos severos en estudios posteriores que muestran que las diferencias en las conexiones nerviosas entre los sexos desaparecen cuando, en las comparaciones, se toma en cuenta el tamaño del cerebro $(6,18,32,34)$.

\section{La neuroética contra el neurosexismo}

Frente a la moda actual por las neurociencias, sus repercusiones en la sociedad y el riesgo de instrumentalización fuera del campo científico, la reflexión ética es esencial $(4,5,35,44)$. La «neuroética» constituye un nuevo campo de investigación en un número creciente de universidades a nivel internacional. En 2010, nació la red «Neuro Genderings» que reúne a investigadores de 
varios países (Australia, Alemania, Austria, Canadá, Estados Unidos, Francia, Italia, Suecia, Suiza) y de diferentes disciplinas: neurociencias, filosofía, sociología, estudios de género, ciencia y tecnología (https://en.wikipedia. org/wiki/The_NeuroGenderings_Network). El objetivo de la red es defender una ética en la producción de conocimiento sobre sexo, género y sexualidad a la luz de los problemas ideológicos y sociopolíticos planteados por la investigación.

En el contexto actual donde las tesis esencialistas resurgen para atacar los estudios de género, es crucial que los biólogos se comprometan junto con las humanidades y las ciencias sociales a desafiar las evidencias falsas que desearían que el orden social sea un reflejo del orden biológico. La cuestión de fondo no es la de las diferencias más o menos marcadas entre los cerebros de mujeres y hombres, sino la del origen de estas diferencias. Pensar en nuestras diferencias a la luz de la plasticidad cerebral hace que el argumento de la naturaleza sea totalmente obsoleto. Abordar los prejuicios esencialistas de frente es esencial para combatir los estereotipos, tomar medidas políticas y construir juntos una cultura de igualdad.

\section{Referencias bibliográficas}

1. Angeles-Han S, Yeh S (2012) Prevention and management of cataracts in children with juvenile idiopathic arthritis-associated uveitis. Curr Rheumatol Rep, 141: 42-9.

2. Braddick O, John Atkinson J (2011) Development of human visual function. Vision Res. , 51:1588-609.

3. Chang Y. (2014) Reorganization and plastic changes of the human brain associated with skill learning and expertise. Front Hum Neurosci., 8-35 : 1-7

4. Charmak B, Moutaud B (2014) Neurosciences et Société, Paris: Armand Colin.

5. Choudhury S, Nagel S, Slaby J (2009) Critical neuroscience: Linking neuroscience and society through critical practice. Biosocieties, 4: 61-77.

6. Coupé P et al. (2017) Towards a Unified Analysis of Brain Maturation and Aging across the Entire Lifespan: A MRI Analysis. Human Brain Mapping, 38: 5501-18.

7. Dean DC et al. (2017) "Investigation of brain structure in the 1-month infant". Brain Structure and Function, 223 1953-1970

8. Dean DC et al (2018) Correction to: Investigation of brain structure in the 1-month infant. Brain Structure and Function, 223: 3007-3009.

9. Fausto-Sterling A (2000) Sexing the Body: Gender Politics and the Construction of Sexuality. New York: Basic Books.

10. Fausto-Sterling A, Garcia C, Lamarre M (2012) Sexing the baby: Part 1 . What do we really know about sex differentiation in the first three years of life? Social Science \& Medecine, 74: 1684-92.

11. Fillod O (2014) Le connectome et la circulation circulaire des stéréotypes de genre:http://allodoxia.blog.lemonde.fr/

12. Fine C (2010) Delusions of Gender: How Our Minds, Society, and Neurosexism Create Difference. New York: Norton.

13. Fine C (2017) Testosterone Rex: Myths of Sex, Science, and Society. New York: Norton.

14. Fine C (2013) Is there neurosexism in functional neuroimaging investigations of sex differences? Neuroethics, 6:369-409.

15. Giedd JN et al. (2012) Magnetic resonance imaging of male/ female differences in human adolescent brain anatomy. Biology of Sex Differences, 3:19-28.

16. Gilmore JH et al., (2012) Longitudinal Development of Cortical and Subcortical Gray Matter from Birth to 2 Years. Cerebral Cortex, $22: 2478-2485$
17. Gould S J (1996) Mismeasure of Man. New York: Norton \& Company.

18. Hänggi J et al. (2014) The hypothesis of neuronal interconnectivity as a function of brain size: a general organization principle of the human connectome. Frontiers in human neuroscience, 8: 3-16.

19. Harenski C, Kiehl K (2009) Gender differences in neural mechanisms underlying moral sensitivity. SCAN, 3: 313-321.

20. Héritier F (1996) Masculin/Féminin. Paris: Odile Jacob.

21. Illes J et al. (2006) ELSI priorities for brain imaging. American Journal of Bioethics, 6: 24-31.

22. Ingalhalikar $M$ et al. (2014) Sex differences in the structural connectome of the humaun brain". Proc Natl Acad Sci U S A, 111: 823-8.

23. Jancke L et al. (2015) Size, sex, and the aging brain. Human brain mapping, $36: 150-69$

24. Joel D et al. (2015) Sex beyond the genitalia: The human brain mosaic. Proceedings of the National Academy of Sciences, 112: $15468-73$.

25. Joel D et al. (2018) Analysis of Human Brain Structure Reveals that the Brain "Types" Typical of Males Are Also Typical of Females, and Vice Versa. Frontiers in Human Neuroscience, 12:399.

26. Jordan-Young R (2010) Brain Storm: The Flaws in the Science of Sex Differences. Cambridge. Harvard: Harvard University Press.

27. Jordan-Young R (2012) Hormones, context, and "Brain Gender": A review of evidence from congénital adrenal hyperplasia. Social Science \& Medicine, 74 : 1738-1744.

28. Jordan-Young R, Karkazis K (2019) Testosterone: An Unauthorized Biography. Harvard: Harvard University Press.

29. Kaiser A et al. (2009) On sex/gender related similarities and differences in fMRI language research. Brain Research Reviews, 61: 49-59.

30. Kaiser A (2015) Re-conceptualizing "sex" and "gender" in the human brain. Z Psychol. 220: 130-136

31. Kraus C, Gardey D, Löwy I (2000) "La bicatégorisation par sexe à l'épreuve de la science", in L'invention du naturel: les sciences et la fabrication du féminin et du masculin. Paris: Archives Contemporaines.

32. Luders E, Toga AW, Thompson PM (2014) Why size matters: Differences in brain volume account for apparent sex differences in callosal anatomy. Neuroimage, 84: 820-824. 
33. May A (2011) Experience-dependent structural plasticity in the adult human brain. Trends in Cognitive Sciences, 15: $475-82$.

34. Pintzka C et al. (2015) Marked effects of intracranial volume correction methods on sex differences in neuroanatomical structures: a HUNT MRI study. Front. Neurosci. Switz. 9:238.

35. Racine E et al. (2010) Contemporary neuroscience in the media. Social Science \& Medicine, 71: 725-733.

36. Rippon et al. (2014) Recommendations for sex/Gender Neuroimaging Research: Key Principles and Implications for Research Design, Analysis, and Interpretation. Frontiers in Human Neuroscience 8: 650- 655.

37. Rippon G (2019) The Gendered Brain: The New Neuroscience That Shatters The Myth Of The Female Brain. London: Bodley Head.

38. Rose S (2006) Lifelines: Biology, freedom, determinism. New York: Vintage Books.
39. Sampaio-Baptista C, Johansen-Berg H (2017) White Matter Plasticity in the Adult Brain. Neuron, 96:1239-1248.

40. Sanchis-Segura et al. (2019) Sex differences in gray matter volume: how many and how large are they really? Biology of Sex Differences, 10:32.

41. Schmitz S, Grit Hoppner G (2014) Neurofeminism and Feminist Neurosciences: A Critical Review of Contemporary Brain Research. Frontiers in Human Neuroscience 8: 1-10.

42. Vidal C (2012) The Sexed Brain: Between Science and Ideology. Neuroethics, 5: 295-303.

43. Vidal C (2015) Nos cerveaux, tous pareils, tous différents! Paris: Belin.

44. Vidal C, Benoit-Browaeys D (2015) Cerveau, Sexe et Pouvoir. Paris: Belin.

45. Vidal C (2019) Nos cerveaux resteront-ils humains? Paris: Le Pommier. 